\title{
Effects of endoplasmic reticulum stress on autophagy and apoptosis of human leukemia cells via inhibition of the PI3K/AKT/mTOR signaling pathway
}

\author{
LI-JUAN LI, YE CHAI, XIAO-JIA GUO, SONG-LIN CHU and LIAN-SHENG ZHANG \\ Department of Hematology, Lanzhou University Second Hospital, Lanzhou, Gansu 730000, P.R. China
}

Received December 29, 2016; Accepted December 18, 2017

DOI: $10.3892 / \mathrm{mmr} .2018 .8840$

\begin{abstract}
The present study aimed to explore the regulatory effects of endoplasmic reticulum stress (ERS) on the phosphoinositide 3-kinase (PI3K)/AKT serine/threonine kinase 1 (AKT)/mammalian target of rapamycin (mTOR) signaling pathway, and its subsequent effects on autophagy and apoptosis of human leukemia cells. Human leukemia cells were cultured and treated with various concentrations of tunicamycin for 0, 24, 48, 72 and $90 \mathrm{~h}$. Subsequently, human leukemia cells were assigned into the ER activation group, which was treated with $100 \mathrm{ng} / \mathrm{ml}$ tunicamycin, the ER activation + TO901317 (PI3K inhibitor) group, and the control group. An MTT assay was conducted to detect cell proliferation. In addition, a monodansylcadaverine (MDC) assay was used to detect the formation of autophagosomes and Annexin V-fluorescein isothiocyanate/propidium iodide double staining was used to examine cell apoptosis. Western blotting was performed to detect the expression levels of 78-kDa glucose-regulated protein (GRP78), phosphorylated (p)-protein kinase R-like endoplasmic reticulum kinase (PERK), p- $\alpha$ subunit of eukaryotic initiation factor 2 (eIF2 $\alpha$ ), microtubule-associated protein 1A/1B-light chain 3 (LC3), caspase-3, CCAAT-enhancer-binding protein homologous protein (CHOP), PI3K, AKT and mTOR. Treatment with $100 \mathrm{ng} / \mathrm{ml}$ tunicamycin for $72 \mathrm{~h}$ was considered the optimal condition for further experiments. Compared with in cells prior to treatment, human leukemia cells treated with tunicamycin exhibited increased expression of p-PERK, p-eIF2 $\alpha$ and GRP78 after $72 \mathrm{~h}(\mathrm{P}<0.05)$. In addition, the expression levels of mTOR, AKT and PI3K were decreased in the ER activation group compared with in the control and ER activation
\end{abstract}

Correspondence to: Dr Lian-Sheng Zhang, Department of Hematology, Lanzhou University Second Hospital, 82 Cuiyingmen Road, Chengguan, Lanzhou, Gansu 730000, P.R. China

E-mail: zhangliansheng953@126.com

Key words: endoplasmic reticulum stress, phosphoinositide 3-kinase/AKT serine/threonine kinase 1/mammalian target of rapamycin signaling pathway, human leukemia cells, autophagy, apoptosis
+ TO901317 groups $(\mathrm{P}<0.05)$. Compared with in the control group, cell proliferation was inhibited and MDC fluorescence intensity was increased in the ER activation group $(\mathrm{P}<0.05)$. Furthermore, compared with in the control and ER activation + TO901317 groups, western blotting indicated that the expression levels of LC3-II were increased in the ER activation group $(\mathrm{P}<0.05)$. The apoptotic rate was also higher in the ER activation group compared with in the control group $(\mathrm{P}<0.05)$, and caspase-3 and CHOP expression was elevated in the ER activation group $(\mathrm{P}<0.05)$. These findings indicated that ERS may induce autophagy and apoptosis of human leukemia cells via inhibiting the PI3K/AKT/mTOR signaling pathway.

\section{Introduction}

Leukemia refers to a heterogeneous group of hematopoietic malignancies that includes four major subtypes: Acute lymphoblastic leukemia, acute myeloid leukemia (AML), chronic lymphocytic leukemia and chronic myeloid leukemia (1). Leukemia is considered the most common childhood cancer; however, it does affect people of all ages, making it a major cause of morbidity and mortality (2). With $>11,000$ people diagnosed with AML each year, $75 \%$ of patients succumb to leukemia within 5 years worldwide (3). In addition, males are more likely to develop leukemia than females, and individuals in developed regions are more likely to develop leukemia than individuals in less developed regions (4). There are numerous risk factors associated with leukemia, including genetic disorders caused by aberrant chromosomes, human T cell leukemia virus, previous long-term exposure to radiation and myelodysplastic syndrome (5). Leukemia cells are white blood cells that are not fully developed and exhibit an abnormally large quantity in leukemia, which are fundamentally necessary to sustain leukemia (5). Previous studies have indicated that inducing autophagy and apoptosis of leukemia cells serves as a feasible therapy for leukemia $(6,7)$. In recent years, efforts have been made to analyze the basic cellular and molecular biology of leukemia, and have demonstrated that complex signaling pathways are involved in leukemia (8); therefore, disrupting these signaling pathways to induce autophagy and apoptosis of leukemia cells may be considered an appealing target for the treatment of leukemia.

The phosphoinositide 3-kinase (PI3K)/protein kinase B $(\mathrm{AKT}) / \mathrm{mammalian}$ target of rapamycin (mTOR) pathway is 
a prototypic survival pathway (9), which has been reported to serve a crucial role in cell growth, proliferation and survival, not only under physiological circumstances but also in numerous tumor cells (10). Previous studies have revealed an association between leukemia and aberrant activation of the PI3K/Akt/mTOR signaling pathway $(11,12)$. The endoplasmic reticulum (ER) is a major site in the cell, which has a role in protein folding and trafficking, and is responsible for numerous cellular functions (13). ER stress (ERS) results from discrepancies between the demand for and the capacities of ER functions (14). Previous studies have indicated that ERS is closely associated with the apoptotic response of human leukemia cells $(15,16)$. However, the mechanism underlying how ERS suppresses the progression of disease in an efficient manner remains unknown. Consequently, the present study explored the effects of ERS on regulation of the $\mathrm{PI} 3 \mathrm{~K} / \mathrm{AKT} / \mathrm{mTOR}$ signaling pathway, and on autophagy and apoptosis of human leukemia cells, with the aim of introducing a novel target for the treatment of leukemia.

\section{Materials and methods}

Preparation and culture of leukemia cells. The present study was approved by the ethics committee of Lanzhou University Second Hospital (Lanzhou, China). Leukemia cells were derived from routine blood samples collected from patients at the Third Affiliated Hospital of Xiangya (Changsha, Hunan, China). All patients provided written informed consent. The patients all underwent clinical hemograms and myelograms, and were diagnosed with leukemia (17). Peripheral blood (white blood cells $>2 \times 10^{10} / 1 ; 3-5 \mathrm{ml}$ ) or bone marrow fluid (1-1.5 ml) samples were extracted from the patients. The inner wall of a needle tube was wetted with $0.2 \mathrm{ml}$ of aseptic heparin sodium anticoagulant ( $2 \mathrm{~g} / \mathrm{l})$ prior to being used to take bone marrow (or peripheral blood). Mononuclear cells were isolated using Hypaque-Ficoll solution. After being washed three times with serum-free RPMI 1640 (11875093; Thermo Fisher Scientific, Inc., Waltham, MA, USA), the cells were suspended in Iscove's modified Dulbecco's media (12440046; Thermo Fisher Scientific, Inc.) to obtain a $2 \times 10^{7} / \mathrm{ml}$ cell suspension. Subsequently T and B lymphocytes were separated, nylon cotton was used to remove the $\mathrm{T}$ cells and a leukemia cell suspension was obtained. The survival rate of mononuclear cells was $>95 \%$. The obtained cells were cultured in Dulbecco's modified Eagle's medium (DMEM) (11320082; Thermo Fisher Scientific, Inc.) containing 10\% fetal bovine serum, $100 \mu \mathrm{g} / \mathrm{ml}$ streptomycin and $100 \mathrm{U} / \mathrm{ml}$ penicillin in a $37^{\circ}$ incubator containing $5 \% \mathrm{CO}_{2}$. Once cell density reached $85 \%$, the cells were digested with $0.5 \%$ trypsin and $0.02 \%$ EDTA and were eluted with PBS. After 3 days, the medium was changed and subculture was performed at 1:3-1:6. The leukemia cells used in the subsequent experiments were in the logarithmic growth phase.

Cell drug toxicity test and grouping. The cells in logarithmic growth phase were inoculated into a 96 -well plate at $2 \times 10^{5}$ cells/ml and $100 \mu \mathrm{l}$ fresh DMEM was added to each well for overnight culture. Following treatment of human leukemia cells with $25,50,100,200$ or $500 \mathrm{ng} / \mathrm{ml}$ tunicamycin (Sigma-Aldrich; Merck Millipore KGaA, Darmstadt,
Germany) for 24, 48, 72 and $96 \mathrm{~h}$, various indicators were detected to determine the optimal concentration and duration of tunicamycin treatment. In the subsequent experiments, leukemia cells were divided into the following three groups: The ER activation group, in which cells were treated with the optimal concentration of tunicamycin $(100 \mathrm{ng} / \mathrm{ml}$ tunicamycin for $72 \mathrm{~h}$ ); the ER activation + TO901317 group, in which cells were treated with the optimal concentration of tunicamycin $+10 \mu \mathrm{mol} / \mathrm{l} \mathrm{PI} 3 \mathrm{~K}$ activator TO901317; and the control group, which consisted of untreated cells.

MTT assay. The cells in logarithmic growth phase were inoculated into a 96-well plate at $2 \times 10^{5}$ cells $/ \mathrm{ml}$ and $100 \mu 1$ fresh DMEM was added to each well for overnight culture. Following the removal of the medium, $100 \mu \mathrm{l}$ fresh medium and $20 \mu 10.5 \%$ MTT solution were added to each well for $3 \mathrm{~h}$ at $37^{\circ} \mathrm{C}$. Subsequently, the medium was removed and $100 \mu \mathrm{l}$ dimethyl sulfoxide was added to each well, and the plate was oscillated at a low speed for 15 min to fully dissolve the crystals. Finally, an enzyme-linked immunometric meter was used to measure the optical density value of each well at $490 \mathrm{~nm}$; three duplicated wells were set up for each group. The MTT assay was used to measure and calculate cell proliferation in each group.

Monodansylcadaverine (MDC) staining. The leukemia cells were inoculated into a 96-well plate and $100 \mu \mathrm{l}$ fresh DMEM was added to each well. The cells were incubated at $37^{\circ} \mathrm{C}$ in an atmosphere containing $5 \% \mathrm{CO}_{2}$ and saturated humidity for $24 \mathrm{~h}$. The cells were collected and the cell concentration adjusted to $10^{6}$ cells $/ \mathrm{ml}$. They were washed twice with PBS. Subsequently, the cells were incubated with $0.05 \mathrm{mM} \mathrm{MDC}$ at $37^{\circ} \mathrm{C}$ for $1 \mathrm{~h}$ and were observed under an inverted fluorescence microscope (Leica DMI 4000B; Leica Microsystems GmbH, Wetzlar, Germany). The fluorescence intensity was calculated as follows: Fluorescence intensity $(\%)=($ fluorescence intensity of treatment groups-control group)/control group x100.

Annexin V-fluorescein isothiocyanate (FITC)/propidium iodide (PI) double staining. The leukemia cells were inoculated into a 96-well plate and $100 \mu \mathrm{l}$ fresh DMEM was added to each well. The cells were incubated at $37^{\circ} \mathrm{C}$ in an atmosphere containing $5 \% \mathrm{CO}_{2}$ and saturated humidity for $24 \mathrm{~h}$. The cells were collected and the cell concentration adjusted to $10^{6}$ cells $/ \mathrm{ml}$. They were washed twice with PBS and centrifuged at $1,000 \times \mathrm{g}$ for $3 \mathrm{~min}$. Subsequently, $500 \mu \mathrm{l}$ binding buffer was added and mixed gently, following which $5 \mu \mathrm{l}$ Annexin V-FITC and $5 \mu \mathrm{l}$ PI were added (K201-100; BioVision, Inc., Milpitas, CA, USA). The cells were incubated in the dark for 10 min and flow cytometry (BD Biosciences, Franklin Lakes, NJ, USA) and CellQuest Pro software version 5.1 (BD Biosciences) were used to detect cell apoptotic rate. The results were analyzed as follows: The lower left quadrant consisted of normal cells, the lower right quadrant consisted of early apoptotic cells, the upper right quadrant consisted of late apoptotic cells and the upper left quadrant consisted of dead cells.

Western blotting. Leukemia cells were treated with the optimal concentration of tunicamycin and were then inoculated into a 
6 -well plate at $1.5 \times 10^{6}$ cells/well for $24 \mathrm{~h}$. Subsequently, the cells were washed three times with ice-cold PBS and protein was extracted from the cleaved cells using RIPA cell lysis solution (BB-3209; BestBio, Co., Shanghai, China). Protein concentration was measured using a Bradford kit (Thermo Fisher Scientific, Inc.), according to the manufacturer's protocol. The proteins $(20-30 \mu \mathrm{g})$ were separated by $10 \%$ SDS-PAGE and were then transferred to a nitrocellulose membrane. The membrane was washed with Tris-buffered saline containing $0.1 \%$ Tween (TBST) and was blocked with 5\% skim milk powder at room temperature for $1 \mathrm{~h}$. Subsequently, the membrane was incubated overnight at $4^{\circ} \mathrm{C}$ with the following primary antibodies: Rabbit polyclonal antibodies: Anti-caspase-3 (ab13847; 1:500; Abcam, Cambridge, UK), anti-mTOR (ab2732; 1:2,000; Abcam), anti-AKT (ab8805; 1:500; Abcam), anti-phosphorylated (p)-protein kinase R-like endoplasmic reticulum kinase (PERK; E19-7579-1; 1:1,000, EnoGene Biotech Co., Ltd., New York, NY, USA), anti-p- $\alpha$ subunit of eukaryotic initiation factor 2 (elF2 $\alpha$; ab227593; 1:1,000; Abcam), anti-microtubule-associated protein 1A/1B-light chain 3 (LC3; ab128025; 1:2,000; Abcam) and anti-78-kDa glucose-regulated protein (GRP78; ab21685; 1:1,000; Abcam), rabbit antibody Anti-PI3K (ab40776; 1:2,000; Abcam). Following washing with TBST, the membrane was incubated with a secondary antibody sheep anti-rabbit IgG (Cell Signaling Technology, Inc., Danvers, MA, USA) for $1 \mathrm{~h}$ at $37^{\circ} \mathrm{C}$. Finally, the membrane was visualized using electrochemiluminescence (ECL) (Beijing Solarbio Science $\&$ Technology Co., Ltd., Beijing, China) for $3 \mathrm{~min}$. Then the membrane was put into the Gel imaging system instrument (Bio-Rad Laboratories, Inc., Hercules, CA, USA), and $400 \mu \mathrm{l}$ ECL hypersensitive luminescence (Beijing Solarbio Science $\&$ Technology Co., Ltd.) added and developed for $2 \mathrm{~min}$. The relative expression of the target protein=gray value of target protein band/gray value of internal reference band ( $\beta$-actin). The experiment was repeated 3 times).

Statistical analysis. All experiments were repeated at least three times under the same conditions. Statistical analyses were conducted using SPSS 21.0 (IBM Corp., Armonk, NY, USA). Measurement data were expressed as the mean \pm standard deviation, enumeration data were expressed as a percentage. Differences between two groups were analyzed using Student's t-test, and differences among multiple groups were analyzed by one-way analysis of variance (ANOVA) and LSD analysis. Cell growth conditions following treatment with various concentrations for various time points were assessed by repeated measures ANOVA. $\mathrm{P}<0.05$ was considered to indicate a statistically significant different.

\section{Results}

ERS is induced by tunicamycin. The results of an MTT assay and flow cytometry demonstrated that cell survival rate was decreased as tunicamycin concentration and treatment duration increased. Specifically, when cells were treated with $100 \mathrm{ng} / \mathrm{ml}$ tunicamycin for $72 \mathrm{~h}$, the survival rate of human leukemia cells was $57.68 \pm 3.12 \%$. This concentration and duration of tunicamycin treatment was used in subsequent experiments (Fig. 1A).
After the leukemia cells were treated with $100 \mathrm{ng} / \mathrm{ml}$ tunicamycin for $72 \mathrm{~h}$, the results of a western blot analysis demonstrated that the expression levels of p-PERK, p-eIF2 $\alpha$ and the ER molecular chaperone GRP78 were significantly increased compared with in cells prior to treatment $(\mathrm{P}<0.05$; Fig. 1B). These findings indicated that ER activation was induced in response to treatment with $100 \mathrm{ng} / \mathrm{ml}$ tunicamycin for $72 \mathrm{~h}$.

Effects of ERS on PI3K/AKT/mTOR signaling pathway-associated protein expression. Following treatment of the leukemia cells with $100 \mathrm{ng} / \mathrm{ml}$ tunicamycin for $72 \mathrm{~h}$, the results of a western blot analysis demonstrated that compared with in the control group, the expression levels of key proteins in the PI3K/AKT/mTOR signaling pathway exhibited no significant difference in the ER activation + TO901317 group $(\mathrm{P}>0.05)$. However, compared with in the control and ER activation + TO901317 groups, the expression levels of mTOR, AKT and PI3K were significantly decreased in the ER activation group $(\mathrm{P}<0.05$; Fig. 2$)$, which indicated that ERS may inhibit the PI3K/AKT/mTOR signaling pathway.

Effects of ERS on cell survival rates. Observation under a microscope demonstrated that, following treatment of leukemia cells with $100 \mathrm{ng} / \mathrm{ml}$ tunicamycin for $72 \mathrm{~h}$, cells in the ER activation and ER activation + TO901317 groups exhibited shrinkage and cracking, and the number of cells markedly decreased while no obvious difference between the ER activation + TO901317 group and control group was observed (Fig. 3A). The results of an MTT assay also revealed that compared with in the control group, cell viability was significantly inhibited in the ER activation group $(\mathrm{P}<0.05)$. Although cell viability was decreased in the ER activation + TO901317 group, there was no significant difference compared with in the control group ( $\mathrm{P}>0.05$; Fig. $3 \mathrm{~B}$ ), which suggested that activation of PI3K may reduce the inhibitory effects of ERS on the viability of human leukemia cells.

Effects of ERS on autophagy in leukemia cells. Increased MDC fluorescence intensity indicates that cell autophagy is increased. Compared with in the control group, MDC intensity in the ER activation + TO901317 group was slightly increased; however, there was no significant difference $(P>0.05)$. MDC fluorescence intensity in was significantly increased the ER activation group $(\mathrm{P}<0.05$; Fig. $4 \mathrm{~A})$. The formation of autophagosomes is positively associated with the expression of LC3-II. The results of a western blot analysis demonstrated that compared with in the control and ER activation + TO901317 groups, the expression levels of LC3-II were significantly increased in the ER activation group ( $\mathrm{P}<0.05$; Fig. $4 \mathrm{~B})$, which indicated that PI3K activation inhibited the occurrence of autophagy, suggesting that ERS-induced autophagy of leukemia cells may be caused by inhibiting the PI3K/AKT/mTOR signaling pathway.

Effects of ERS on apoptosis of leukemia cells. The results of a flow cytometric analysis demonstrated that compared with in the control group, the apoptotic rates of the other two groups were increased; the apoptotic rate in the ER activation group was significantly increased $(\mathrm{P}<0.05 ;$ Fig. $5 \mathrm{~A})$. The results of a 
A

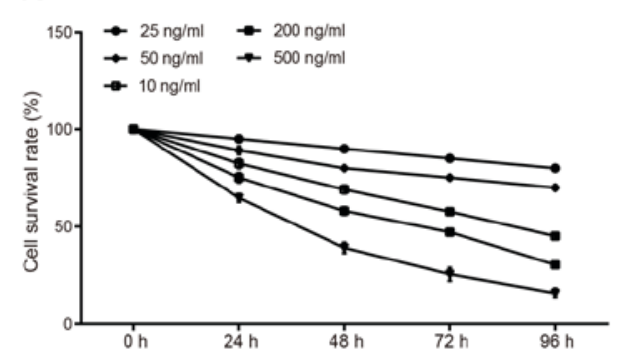

B

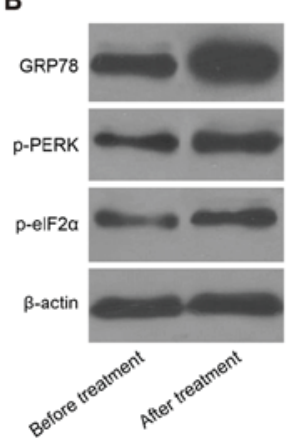

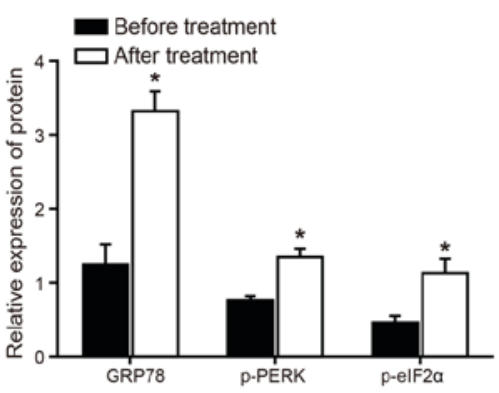

Figure 1. Endoplasmic reticulum stress is induced by tunicamycin. (A) Cell survival at different time points after treatment with various concentrations of tunicamycin. (B) Protein expression levels following treatment of cells with $100 \mathrm{ng} / \mathrm{ml}$ tunicamycin for $72 \mathrm{~h}$. $\mathrm{P}<0.05$, compared with before tunicamycin treatment. eif2, $\alpha$ subunit of eukaryotic initiation factor 2; GRP78, 78-kDa glucose-regulated protein; p-, phosphorylated; PERK, protein kinase R-like endoplasmic reticulum kinase.
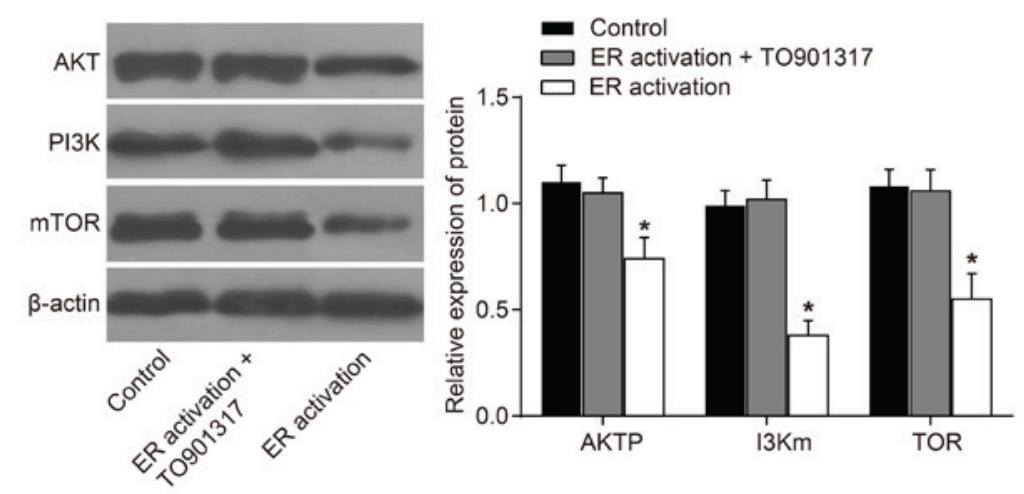

Figure 2. Effects of ER stress on the PI3K/AKT/mTOR signaling pathway among the three groups. ${ }^{\text {P }}<0.05$ vs. the control group. AKT, AKT serine/threonine kinase 1; ER, endoplasmic reticulum; mTOR, mammalian target of rapamycin; PI3K, phosphoinositide 3-kinase.

A

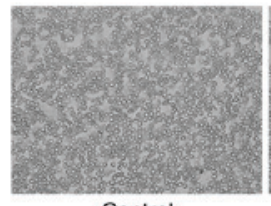

Control

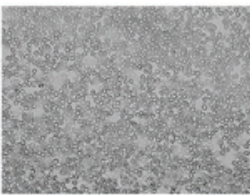

ER activation + T0901317

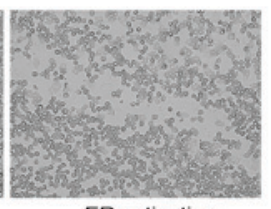

ER activation

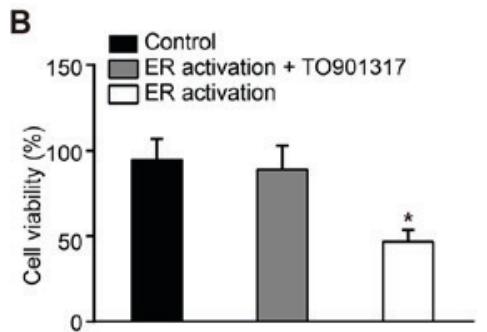

Figure 3. Alterations in cell viability in leukemia cells among the three groups. (A) Inverted microscopy (magnification, x200) and (B) an MTT assay confirmed that cell viability was reduced in the ER activation group. ${ }^{*} \mathrm{P}<0.05$ vs. the control group. ER, endoplasmic reticulum.

western blot analysis indicated that in the ER activation group, the apoptosis-associated key factor caspase-3 was activated and the expression levels of CHOP were significantly increased compared with in the control group $(\mathrm{P}<0.05)$, thus suggesting that apoptosis occurred in this cell group. No significant differences were observed in the expression of caspase-3 and CHOP between the ER activation + TO901317 group and the control group ( $\mathrm{P}>0.05$; Fig. $5 \mathrm{~B})$, thus suggesting that activation of PI3K could inhibit the apoptosis of human leukemia cells. In addition, these results indicated that ERS may induce apoptosis of human leukemia cells by inhibiting the PI3K/AKT/mTOR signaling pathway.

\section{Discussion}

Leukemia affects individuals of all ages and is therefore a major cause of morbidity and mortality worldwide (2). Due to the strenuous efforts made by researchers, understanding the cellular and molecular biology of leukemia has markedly advanced, and numerous signaling pathways have been reported to be involved in its pathogenesis, including the PI3K/AKT/mTOR pathway $(8,11)$. However, the mechanism underlying how these pathways function remains to be determined; therefore, studies are required to explore the specifics behind complex pathways. 

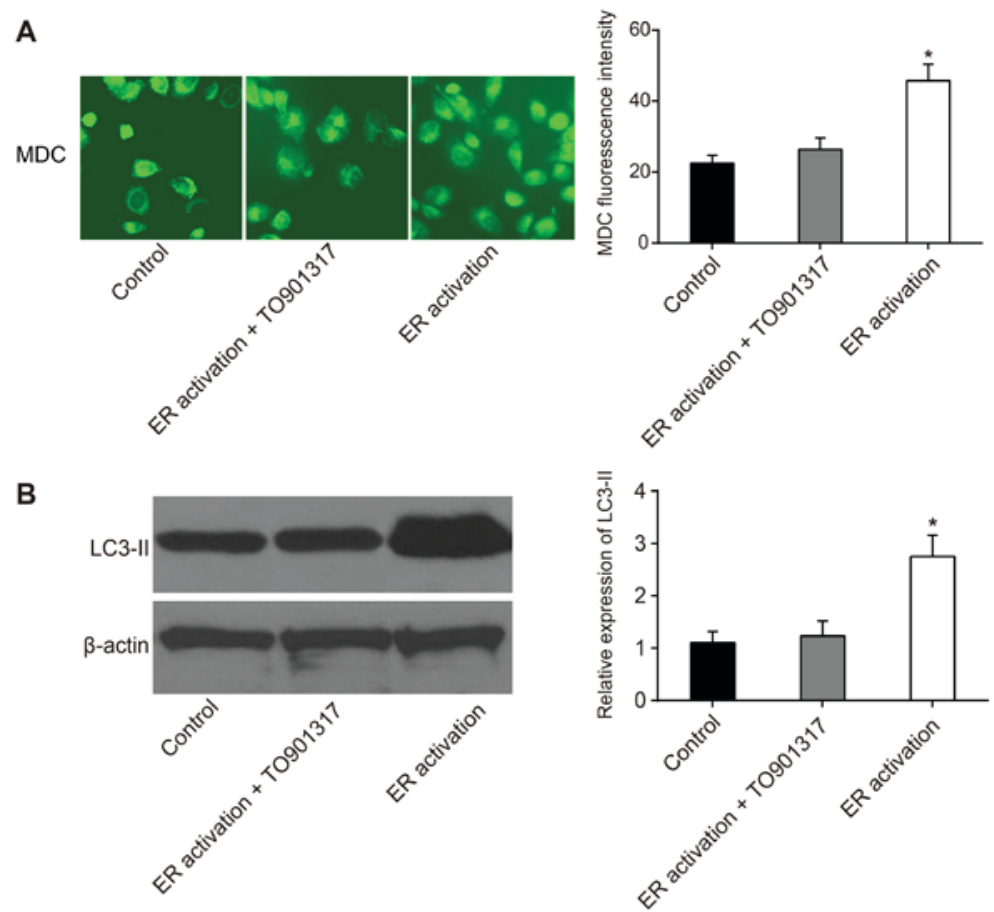

Figure 4. Alterations in cell autophagy caused by ERS among the three groups. Effects of ERS on (A) MDC fluorescence intensity (magnification, x400) and (B) protein expression levels of LC3-II, as detected by western blotting. "P $<0.05$ vs. the control group. ERS, endoplasmic reticulum stress; LC3, microtubule-associated protein 1A/1B-light chain 3; MDC, monodansylcadaverine.
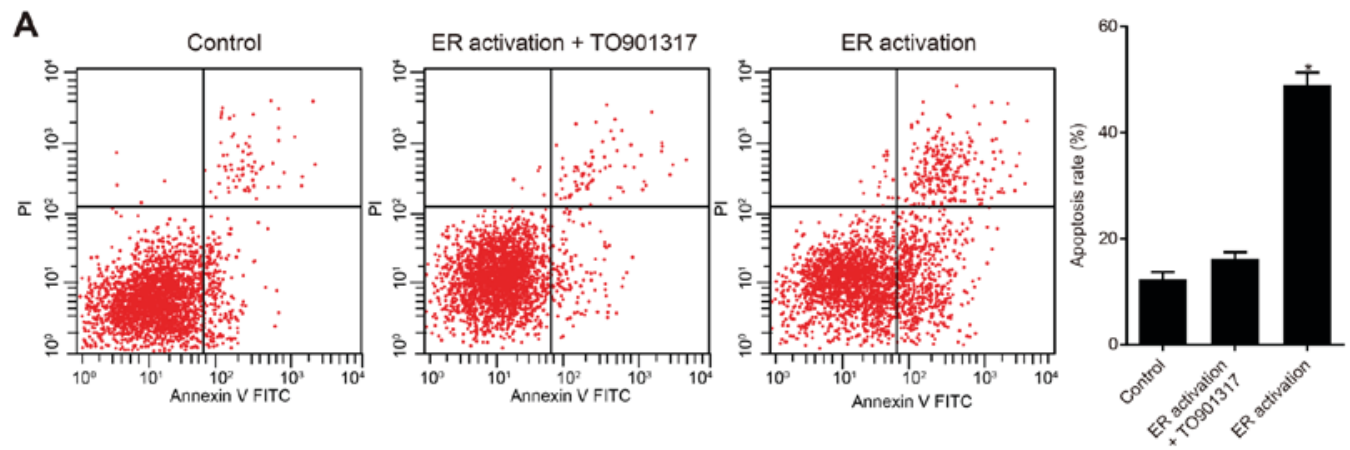

B
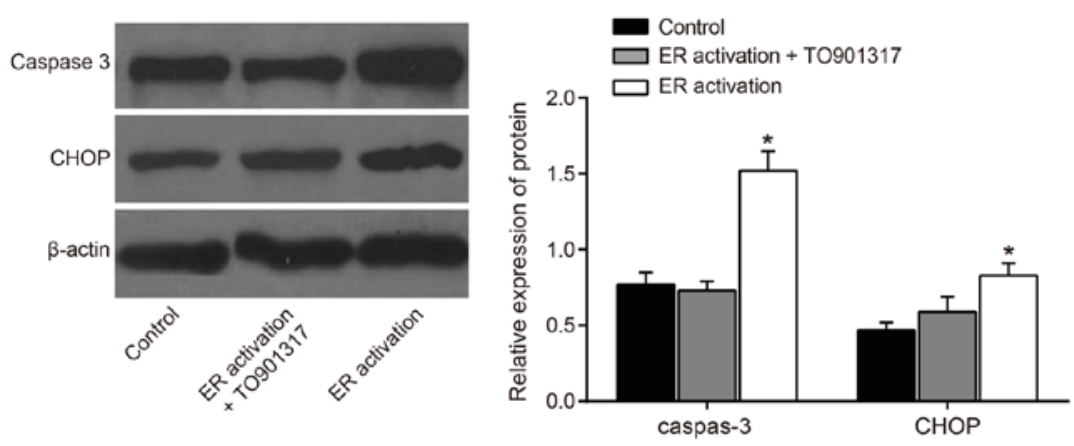

Figure 5. Effects of ERS on apoptosis of leukemia cells among the three groups. (A) Alterations in the apoptotic rate of cells undergoing ERS; (B) expression levels of caspase- 3 and CHOP in cells undergoing ERS. " $\mathrm{P}<0.05$ vs. the control group. CHOP, CCAAT-enhancer-binding protein homologous protein; ERS, endoplasmic reticulum stress; FITC, fluorescein isothiocyanate; PI, propidium iodide.

The present study revealed that in the presence of tunicamycin-induced ERS, the expression levels of mTOR, AKT and PI3K were markedly decreased, thus suggesting that ERS may suppress the PI3K/AKT/mTOR signaling pathway. Previous studies have reported that PI3K and its downstream targets AKT and mTOR have an essential role in physiological processes, including cell growth, survival, differentiation and proliferation, as well as in the development 
of malignant disease $(18,19)$. The PI3K/AKT/mTOR pathway works as follows: PI3K, which is upstream of AKT, is responsible for activating AKT, the activated AKT proceeds to phosphorylate target molecules, including mTOR, which is a master regulator of protein translation (11). In addition, phosphatase and tensin homolog (PTEN), which is a well-characterized human tumor suppressor gene, has been reported to possess a high frequency of abnormalities in leukemia and has also been established as an antagonist of the PI3K/AKT/mTOR pathway $(9,20)$. Therefore, it may be hypothesized that under ERS, PTEN dephosphorylates and thereby suppresses PI3K, which further disrupts AKT and mTOR activity, thus inducing downregulated expression of mTOR, AKT and PI3K (21). Di Nardo et al conducted experiments on rat hippocampal neurons and revealed that tunicamycin-induced ERS can downregulate AKT and mTOR activity (22), which is consistent with the results of the present study.

The present study also indicated that ERS markedly inhibits the proliferative capacity of leukemia cells, so as to induce autophagy and apoptosis via suppression of the $\mathrm{PI} 3 \mathrm{~K} / \mathrm{AKT} / \mathrm{mTOR}$ signaling pathway. As a process that describes the degradation and recycling of proteins and intracellular components in reaction to starvation or stress, autophagy can often lead to cell death, which is most commonly associated with apoptosis (21). It has previously been reported that ERS causes mitochondrial damage and initiates cell apoptosis, the process of which may be worsened by oxidative stress when accompanied by an mTOR inhibitor (23). mTOR is the catalytic subunit of two biochemically distinct molecular complexes, namely, mTOR complex (MTORC)1 whose activation increases protein synthesis and inhibits autophagy, and mTORC2 whose activation causes additional phosphorylation of AKT and promotes cell survival and proliferation (24). Therefore, since the mTOR pathway initiates the translation of mRNAs that encode proteins required for cell cycle progression, inhibition of the signaling pathway may arrest the development of disease to some extent (25). In this sense, ERS may induce autophagy and apoptosis via the suppression of mTOR (26). Chen et al reported similar results to the present study and identified a causal relationship between ERS and autophagy-mediated apoptosis in human hepatocellular carcinoma cell lines with the aid of PI3K/AKT/mTOR signaling pathway inhibition (27).

In conclusion, by inhibiting the PI3K/AKT/mTOR signaling pathway ERS may induce autophagy and apoptosis of leukemia cells, and may be a potential target in treating leukemia. However, although a role has been identified for the PI3K/AKT/mTOR signaling pathway in autophagy and apoptosis, its specific mechanism requires verification due to the complicated processes involved in the pathway. As a result, further studies are required to investigate the exact mechanism underlying the effects of the PI3K/AKT/mTOR signaling pathway on autophagy and apoptosis in various types of leukemia.

\section{Acknowledgements}

The authors would like to thank all the reviewers and editors who gave assistance and helpful discussions for our manuscript.

\section{Funding}

No funding was received.

\section{Availability of data and materials}

The datasets used and/or analyzed during the current study are available from the corresponding author on reasonable request.

\section{Authors' contributions}

LJL, YC and XJG designed the study. SLC and LSZ collated the data, designed and developed the database, carried out data analyses and produced the initial draft of the manuscript. LJL and YC contributed to drafting and revising the manuscript. All authors read and approved the final submitted manuscript.

\section{Ethics approval and consent to participate}

The present study was approved by the Ethics Committee of Lanzhou University Second Hospital (Lanzhou, China).

\section{Consent for publication}

All patients provided written informed consent.

\section{Competing interests}

The authors declare that they have no competing interests.

\section{References}

1. Xie Y, Davies SM, Xiang Y, Robison LL and Ross JA: Trends in leukemia incidence and survival in the United States (1973-1998). Cancer 97: 2229-2235, 2003.

2. Tower RL and Spector LG: The epidemiology of childhood leukemia with a focus on birth weight and diet. Crit Rev Clin Lab Sci 44: 203-242, 2007.

3. Ali NA, O'Brien JM Jr, Blum W, Byrd JC, Klisovic RB, Marcucci G, Phillips G, Marsh CB, Lemeshow S and Grever MR: Hyperglycemia in patients with acute myeloid leukemia is associated with increased hospital mortality. Cancer 110: 96-102, 2007.

4. Rajabli N, Naeimi-Tabeie M, Jahangirrad A, Sedaghat SM, Semnani S and Roshandel G: Epidemiology of leukemia and multiple myeloma in Golestan, Iran. Asian Pac J Cancer Prev 14: 2333-2336, 2013.

5. Rhomberg LR, Bailey LA, Goodman JE, Hamade AK and Mayfield D: Is exposure to formaldehyde in air causally associated with leukemia?-A hypothesis-based weight-of-evidence analysis. Crit Rev Toxicol 41: 555-621, 2011.

6. Yokoyama T, Miyazawa K, Naito M, Toyotake J, Tauchi T, Itoh M, Yuo A, Hayashi Y, Georgescu MM, Kondo Y, et al: Vitamin K2 induces autophagy and apoptosis simultaneously in leukemia cells. Autophagy 4: 629-640, 2008.

7. Ge J, Liu Y, Li Q, Guo X, Gu L, Ma ZG and Zhu YP: Resveratrol induces apoptosis and autophagy in T-cell acute lymphoblastic leukemia cells by inhibiting Akt/mTOR and activating p38-MAPK. Biomed Environ Sci 26: 902-911, 2013.

8. Bao T, Smith BD and Karp JE: New agents in the treatment of acute myeloid leukemia: A snapshot of signal transduction modulation. Clin Adv Hematol Oncol 3: 287-296, 302, 2005.

9. LoPiccolo J, Blumenthal GM, Bernstein WB and Dennis PA: Targeting the PI3K/Akt/mTOR pathway: Effective combinations and clinical considerations. Drug Resist Updat 11: 32-50, 2008.

10. Martelli AM, Evangelisti C, Chiarini F, Grimaldi C, Manzoli L and McCubrey JA: Targeting the PI3K/AKT/mTOR signaling network in acute myelogenous leukemia. Expert Opin Investig Drugs 18: 1333-1349, 2009. 
11. Ikezoe T, Nishioka C, Bandobashi K, Yang Y, Kuwayama Y, Adachi Y, Takeuchi T, Koeffler HP and Taguchi H: Longitudinal inhibition of PI3K/Akt/mTOR signaling by LY294002 and rapamycin induces growth arrest of adult T-cell leukemia cells. Leuk Res 31: 673-682, 2007.

12. Steelman LS, Abrams SL, Whelan J, Bertrand FE, Ludwig DE, Basecke J, Libra M, Stivala F, Milella M, et al: Contributions of the Raf/MEK/ERK, PI3K/PTEN/Akt/mTOR and Jak/STAT pathways to leukemia. Leukemia 22: 686-707, 2008.

13. Hotamisligil GS: Endoplasmic reticulum stress and the inflammatory basis of metabolic disease. Cell 140: 900-917, 2010.

14. Banhegyi G, Baumeister P, Benedetti A, Dong D, Fu Y, Lee AS, Li J, Mao C, Margittai E, Ni M, et al: Endoplasmic reticulum stress. Ann N Y Acad Sci 1113: 58-71, 2007.

15. Rahmani M, Davis EM, Crabtree TR, Habibi JR, Nguyen TK Dent $\mathrm{P}$ and Grant S: The kinase inhibitor sorafenib induces cell death through a process involving induction of endoplasmic reticulum stress. Mol Cell Biol 27: 5499-5513, 2007.

16. Pae HO, Jeong SO, Jeong GS, Kim KM, Kim HS, Kim SA, Kim YC, Kang SD, Kim BN and Chung HT: Curcumin induces pro-apoptotic endoplasmic reticulum stress in human leukemia HL-60 cells. Biochem Biophys Res Commun 353: 1040-1045, 2007.

17. Haferlach T, Kohlmann A, Schnittger S, Dugas M, Hiddemann W Kern W and Schoch C: Global approach to the diagnosis of leukemia using gene expression profiling. Blood 106: 1189-1198, 2005.

18. Song Q, Han CC, Xiong XP, He F, Gan W, Wei SH, Liu HH, Li L and Xu HY: PI3K-Akt-mTOR signal inhibition affects expression of genes related to endoplasmic reticulum stress. Genet Mol Res 15: 15037868, 2016.

19. Kim HS, Kim TJ and Yoo YM: Melatonin combined with endoplasmic reticulum stress induces cell death via the PI3K/Akt/mTOR pathway in B16F10 melanoma cells. PLoS One 9: e92627, 2014.
20. Gutierrez A, Sanda T, Grebliunaite R, Carracedo A, Salmena L, Ahn Y, Dahlberg S, Neuberg D, Moreau LA, Winter SS, et al: High frequency of PTEN, PI3K, and AKT abnormalities in T-cell acute lymphoblastic leukemia. Blood 114: 647-650, 2009.

21. Kondo Y, Kanzawa T, Sawaya R and Kondo S: The role of autophagy in cancer development and response to therapy. Nat Rev Cancer 5: 726-734, 2005.

22. Di Nardo A, Kramvis I, Cho N, Sadowski A, Meikle L, Kwiatkowski DJ and Sahin M: Tuberous sclerosis complex activity is required to control neuronal stress responses in an mTOR-dependent manner. J Neurosci 29: 5926-5937, 2009.

23. Chen MH, Chiang KC, Cheng CT, Huang SC, Chen YY, Chen TW, Yeh TS, Jan YY, Wang HM, Weng JJ, et al: Antitumor activity of the combination of an HSP90 inhibitor and a PI3K/mTOR dual inhibitor against cholangiocarcinoma. Oncotarget 5: 2372-2389, 2014.

24. Pavlidou A and Vlahos NF: Molecular alterations of PI3K/Akt/mTOR pathway: A therapeutic target in endometrial cancer. ScientificWorldJournal 2014: 709736, 2014.

25. Bjornsti MA and Houghton PJ: The TOR pathway: A target for cancer therapy. Nat Rev Cancer 4: 335-348, 2004.

26. Qin L, Wang Z, Tao L and Wang Y: ER stress negatively regulates AKT/TSC/mTOR pathway to enhance autophagy. Autophagy 6: 239-247, 2010.

27. Chen XL, Fu JP, Shi J, Wan P, Cao H and Tang ZM: CXC195 induces apoptosis and endoplastic reticulum stress in human hepatocellular carcinoma cells by inhibiting the PI3K/Akt/mTOR signaling pathway. Mol Med Rep 12: 8229-8236, 2015. 